PROCEEDINGS OF THE

AMERICAN MATHEMATICAL SOCIETY

Volume 138, Number 3, March 2010, Pages 853-859

S 0002-9939(09)10160-0

Article electronically published on November 10, 2009

\title{
POLYNOMIAL GROWTH OF THE CODIMENSIONS: A CHARACTERIZATION
}

\author{
A. GIAMBRUNO AND S. MISHCHENKO
}

(Communicated by Martin Lorenz)

\begin{abstract}
Let $A$ be a not necessarily associative algebra over a field of characteristic zero. Here we characterize the T-ideal of identities of $A$ in case the corresponding sequence of codimensions is polynomially bounded.
\end{abstract}

\section{INTRODUCTION}

This paper is concerned with not necessarily associative algebras whose sequence of codimensions is polynomially bounded. Let $A$ be an algebra over a field $F$ of characteristic zero and let $\operatorname{Id}(A)$ be the T-ideal of polynomial identities of $A$. If $P_{n}$ is the space of multilinear polynomials in the nonassociative and noncommutative variables $x_{1}, \ldots, x_{n}$, then $P_{n}(A)=\frac{P_{n}}{P_{n} \cap \mathrm{Id}(A)}$ has a natural structure of a module for the symmetric group $S_{n}$. Its character, denoted $\chi_{n}(A)$, is called the $n$-th cocharacter of $A$ and its degree $c_{n}(A)=\operatorname{deg} \chi_{n}(A)$ is the $n$-th codimension of $A$. The purpose of this note is to characterize $\operatorname{Id}(A)$ in case the sequence $\left\{c_{n}(A)\right\}_{n \geq 1}$ is polynomially bounded.

In general the $n$-th codimension is bounded by $n ! C_{n}$ where $C_{n}$ is the $n$-th Catalan number. For associative algebras satisfying a nontrivial polynomial identity, $\left\{c_{n}(A)\right\}_{n \geq 1}$ is exponentially bounded ([8]), but this same conclusion does not hold for Lie algebras $([9])$. Nevertheless overexponential bounds are known for such algebras with nontrivial polynomial identity. It is well-known that for both associative and Lie algebras the sequence of codimensions cannot have intermediate growth (between polynomial and exponential) and the case of polynomial growth has been studied in both cases through a characterization of the corresponding T-ideal as follows (see [1], [5]).

Decompose the $n$-th cocharacter

$$
\chi_{n}(A)=\sum_{\lambda \vdash n} m_{\lambda} \chi_{\lambda},
$$

where $\chi_{\lambda}$ is the irreducible $S_{n}$-character corresponding to the partition $\lambda \vdash n$ with multiplicity $m_{\lambda} \geq 0$. Then, if $A$ is an associative or Lie algebra, $\left\{c_{n}(A)\right\}_{n \geq 1}$ is polynomially bounded if and only if there exists a constant $K \geq 0$ such that

Received by the editors March 9, 2009, and, in revised form, August 6, 2009.

2010 Mathematics Subject Classification. Primary 17A50, 16R10, 16P90; Secondary 20C30.

Key words and phrases. Polynomial identity, cocharacter, codimension.

The first author was partially supported by MIUR of Italy.

The second author was partially supported by RFBR grant 07-01-00080.

(C)2009 American Mathematical Society 
$m_{\lambda}=0$ whenever $\lambda=\left(\lambda_{1}, \ldots, \lambda_{r}\right) \vdash n$ and $n-\lambda_{1}>K$. This result is no longer valid even in the case of Jordan algebras, as will be shown below.

Here we shall give a characterization for general nonassociative algebras. We shall prove that $\left\{c_{n}(A)\right\}_{n \geq 1}$ is polynomially bounded if and only if 1 ) there exists a constant $K$ such that $m_{\lambda}=0$ whenever either $n-\lambda_{1}>K$ or $n-\lambda_{1}^{\prime}>K$, where $\lambda^{\prime}=\left(\lambda_{1}^{\prime}, \ldots, \lambda_{s}^{\prime}\right) \vdash n$ is the conjugate partititon of $\lambda$ and 2) there exist constants $k, C$ such that every polynomial $f \in P_{n}$ can be written $(\bmod \operatorname{Id}(A))$ as a linear combination of $\leq C n^{k}$ polynomials $f_{i}$ and each $f_{i}$ has a fixed arrangement of parentheses. We shall also show by some examples that the conditions of the theorem cannot be weakened.

\section{The General Setting}

Throughout $A$ is a not necessarily associative algebra over a field $F$ of characteristic zero and $F\{X\}$ the free nonassociative algebra on a countable set $X=$ $\left\{x_{1}, x_{2}, \ldots\right\}$. The polynomial identities satisfied by $A$ form a T-ideal $\operatorname{Id}(A)$ of $F\{X\}$ and by the standard multilinearization process, we consider only the multilinear polynomials lying in $\operatorname{Id}(A)$. To this end, for every $n \geq 1$, we set $P_{n}$ to be the space of multilinear polynomials in $x_{1}, \ldots, x_{n}$, and we let the symmetric group $S_{n}$ act on $P_{n}$ by setting $\sigma f\left(x_{1}, \ldots, x_{n}\right)=f\left(x_{\sigma(1)}, \ldots, x_{\sigma(n)}\right)$.

The space $P_{n}(A)=P_{n} /\left(P_{n} \cap \operatorname{Id}(A)\right)$ has an induced structure of an $S_{n}$-module, and we let $\chi_{n}(A)$ be its character, called the $n$-th cocharacter of $A$. By complete reducibility we write

$$
\chi_{n}(A)=\sum_{\lambda \vdash n} m_{\lambda} \chi_{\lambda},
$$

where $\chi_{\lambda}$ is the irreducible $S_{n}$-character corresponding to the partition $\lambda \vdash n$ and $m_{\lambda} \geq 0$ is the corresponding multiplicity (we refer the reader to 3 for an account of this approach).

Now, for a fixed arrangement of the parentheses $T$, let us denote by $P_{n}^{T}$ the subspace of $P_{n}$ spanned by the monomials whose arrangement of parentheses is precisely $T$. Also let $P_{n}^{T}(A)=P_{n}^{T} /\left(P_{n}^{T} \cap \operatorname{Id}(A)\right)$. Then clearly $P_{n}(A)=\sum_{T} P_{n}^{T}(A)$.

Since the $S_{n}$-module $P_{n}^{T}(A)$ is a homomorphic image of $P_{n}^{T} \equiv F S_{n}$, the regular $S_{n}$-representation, it follows that if $\chi_{n}(A)^{T}$ is the $S_{n}$-character of $P_{n}^{T}(A)$, then

$$
\chi_{n}(A)^{T}=\sum_{\lambda \vdash n} m_{\lambda}^{T} \chi_{\lambda}
$$

and $m_{\lambda}^{T} \leq d_{\lambda}=\operatorname{deg} \chi_{\lambda}$. Clearly $m_{\lambda} \leq \sum_{T} m_{\lambda}^{T}$.

For $\lambda=\left(\lambda_{1}, \lambda_{2}, \ldots\right) \vdash n$ we denote by $\lambda^{\prime}=\left(\lambda_{1}^{\prime}, \lambda_{2}^{\prime}, \ldots\right)$ the conjugate partition of $\lambda$. Also, we will write $\lambda \leq \mu$ if and only if $\lambda_{i} \leq \mu_{i}$, for all $i \geq 1$.

We next state a special case of [6, Theorem 2] that we shall need in what follows. In what follows we abbreviate the partition $(a, \ldots, a), b$ parts, as $\left(a^{b}\right)$. Also, for a real number $\alpha,[\alpha]$ denotes the integral part of $\alpha$.

Theorem 1 ([6]). Let $\left\{n_{s}\right\}_{s} \geq 1$ be a sequence of positive integers such that $\lim _{s \rightarrow \infty} n_{s}=+\infty$ and let $\{\lambda(s)\}_{s \geq 1}$ be a sequence of partitions $\lambda(s) \vdash n_{s}$ such that $\lambda(s) \leq\left(a_{s}^{a_{s}}\right)$ where $a_{s}=\left[\frac{n_{s}}{2}\right]$. Then, for any $B$ with $0<B<2$, there exists a constant $N$ such that $\operatorname{deg} \chi_{\lambda(s)}>B^{n_{s}}$, for all $s \geq N$. 


\section{Polynomial growth of the codimensions}

We start with an easy computation of a bound for the degree of an $S_{n}$-character.

Remark 1. For any $\lambda \vdash n$ we have that $\operatorname{deg} \chi_{\lambda}>\frac{(n-2 s)^{s}}{s^{s}}$, where $s=n-\lambda_{1}$ or $s=n-\lambda_{1}^{\prime}$.

Proof. If the $h_{i j}$ 's are the hook numbers of the partition $\lambda$, then by the hook formula (see [4]),

$$
\begin{gathered}
\operatorname{deg} \chi_{\lambda}=\frac{n !}{\prod h_{i j}}>\frac{n !}{s^{s}(n-2 s) ! n(n-1) \cdots(n-s+1)} \\
=\frac{(n-s)(n-s-1) \cdots(n-2 s+1)}{s^{s}}>\frac{(n-2 s)^{s}}{s^{s}} .
\end{gathered}
$$

Next we state and prove the main result of the paper.

Theorem 2. Let $A$ be a not necessarily associative algebra over a field of characteristic zero. Then the sequence of codimensions $c_{n}(A), n=1,2, \ldots$, is polynomially bounded if and only if

(1) there exists an integer $N$ such that for all $n \geq 1, m_{\lambda} \neq 0$ in $\chi_{n}(A)=$ $\sum_{\lambda \vdash n} m_{\lambda} \chi_{\lambda}$ implies $n-\lambda_{1}<N$ or $n-\lambda_{1}^{\prime}<N$;

(2) there exist constants $C, k$ such that for every $n \geq 1$ there exist $m \leq C n^{k}$ arrangements of parentheses $T_{1}, \ldots, T_{m}$ such that every polynomial $\bar{f} \in P_{n}$ can be written as

$$
f \equiv \sum_{i=1}^{m} f_{i} \quad(\bmod \operatorname{Id}(A))
$$

where $f_{i} \in P_{n}^{T_{i}}$.

Proof. Assume that conditions (1) and (2) hold and let $\lambda \vdash n$ be such that $m_{\lambda} \neq 0$ in $\chi_{n}(A)=\sum m_{\lambda} \chi_{\lambda}$. Then $n-\lambda_{1}<N$ or $n-\lambda_{1}^{\prime}<N$ and by the hook formula it is easily deduced that $\operatorname{deg} \chi_{\lambda} \leq a n^{2 N}$, for some constant $a$. Now, for any fixed arrangement $T$ of the parentheses, $\chi_{n}(A)^{T}=\sum m_{\lambda}^{T} \chi_{\lambda}$, and $m_{\lambda} \leq \operatorname{deg} \chi_{\lambda}$, for all $\lambda \vdash n$. Hence it follows that $m_{\lambda}^{T} \leq a n^{2 N}$. This together with condition (2) implies that

$$
c_{n}(A)=\sum_{\lambda \vdash n} m_{\lambda} \operatorname{deg} \chi_{\lambda} \leq \sum_{T} \sum_{\lambda \vdash n} m_{\lambda}^{T} \operatorname{deg} \chi_{\lambda} \leq C_{1} n^{k_{1}},
$$

where $C_{1}=C a^{2}$ and $k_{1}=4 N+k$. We remark that the above argument was used in [1, where only the case $n-\lambda_{1}<N$ was considered.

Suppose now that $c_{n}(A), n=1,2, \ldots$, is polynomially bounded. Fix a permutation $\sigma \in S_{n}$ and, for an arrangement of the parentheses $T$, let $f^{T}$ be the corresponding monomial of $P_{n}$. Let $W_{\sigma}=\operatorname{span}\left\{f^{T} \mid T\right\}$ be the subspace of $P_{n}$ spanned by the monomials $f^{T}$, where $T$ ranges over all arrangements of the parentheses. Then $\operatorname{dim} W_{\sigma}$ is the $n$-th Catalan number. Let $f^{T_{1}}, \ldots, f^{T_{s}}$ be a basis of $W_{\sigma}$ modulo $\operatorname{Id}(A)$. Since by hypothesis $c_{n}(A) \leq a n^{t}$ is polynomially bounded for some constants $a, t$, then $s \leq c_{n}(A) \leq a n^{t}$. It follows that for any fixed arrangement $T$ of the parentheses,

$$
f^{T} \equiv \sum_{r=1}^{s} \alpha_{r} f^{T_{r}} \quad(\bmod \operatorname{Id}(A))
$$


for some $\alpha_{1}, \ldots, \alpha_{s} \in F$. We remark that since $\operatorname{Id}(A)$ is invariant by permutation of the variables, $T_{1}, \ldots, T_{s}$ can be determined so that they do not depend on $\sigma$. In other words the same conclusion holds for any permutation $\tau \in S_{n}$. This implies that condition (2) holds.

Now fix constants $C, k$ such that $c_{n}(A) \leq C n^{k}$, for all $n \geq 1$, and suppose by contradiction that the conclusion (1) of the theorem does not hold. This means that if we consider the set

$$
\begin{gathered}
S=\left\{s \in \mathbb{N} \mid \text { there exists } n_{s} \text { and } \lambda \vdash n_{s} \text { with } m_{\lambda} \neq 0\right. \text { and } \\
\left.n_{s}-\lambda_{1}=s, n_{s}-\lambda_{1}^{\prime} \geq s \text { or } n_{s}-\lambda_{1} \geq s, n_{s}-\lambda_{1}^{\prime}=s\right\},
\end{gathered}
$$

then $S$ contains an infinite number of integers. We consider the infinite sequence $\left\{n_{s}\right\}_{s \in S}$ and we are going to show that there exists $s \in S$ such that $\operatorname{deg} \chi_{\lambda}>$ $C n_{s}^{k}$. This will finish the proof. In fact in this case $c_{n_{s}}(A) \geq m_{\lambda} \chi_{\lambda}>C n_{s}^{k}$, a contradiction.

Now, for any $s \in S$ write $n_{s}=s a_{s}$ and consider the sequence $\left\{a_{s}\right\}_{s \in S}$.

Suppose first that such a sequence is not bounded. Then there exists a subsequence $\left\{a_{s_{i}}\right\}_{i \geq 1}$, such that $\lim _{i \rightarrow \infty} a_{s_{i}}=+\infty$. Recalling that by hypothesis $c_{n}(A) \leq C n^{k}$, for all $n \geq 1$, we have

$$
\begin{gathered}
\lim _{i \rightarrow \infty} \frac{c_{n_{s_{i}}}(A)}{\left(a_{s_{i}}-2\right)^{s_{i}}} \leq \lim _{i \rightarrow \infty} \frac{C n_{s_{i}}^{k}}{\left(a_{s_{i}}-2\right)^{s_{i}}} \\
=C \lim _{i \rightarrow \infty} \frac{s_{i}^{k}}{2^{s_{i}-k}} \cdot \frac{b_{s_{i}}^{k}}{\left(b_{s_{i}}-1\right)^{s_{i}}}=C 2^{k} \lim _{i \rightarrow \infty} \frac{s_{i}{ }^{k}}{2^{s_{i}}} \cdot \frac{b_{s_{i}}^{k}}{\left(b_{s_{i}}-1\right)^{k}} \cdot \frac{1}{\left(b_{s_{i}}-1\right)^{s_{i}-k}}=0,
\end{gathered}
$$

where $a_{s_{i}}=2 b_{s_{i}}$.

Thus there exists $N_{1} \geq 1$ such that for all $i \geq N_{1}$ we have

$$
c_{n_{s_{i}}}(A)<\left(a_{s_{i}}-2\right)^{s_{i}}=\frac{\left(n_{s_{i}}-2 s_{i}\right)^{s_{i}}}{s_{i}^{s_{i}}} .
$$

By Remark 1 we then get that $\operatorname{deg} \chi_{\lambda}>c_{n_{s_{i}}}(A)$, and we are done.

Therefore we may assume that the sequence $\left\{a_{s}\right\}_{s \in S}$ is bounded by some constant $M \geq 1$. Now, since $\operatorname{deg} \chi_{\lambda}=\operatorname{deg} \chi_{\lambda^{\prime}}$, without loss of generality we may assume that for $s \in S, n_{s}-\lambda_{1}=s$ and $n_{s}-\lambda_{1}^{\prime} \geq s$, where $m_{\lambda} \neq 0$.

Suppose first that there exists a subsequence $\left\{a_{s_{i}}\right\}_{i \geq 1}$ such that $2<a_{s_{i}} \leq M$, for all $i \geq 1$. Let $\lambda \vdash n_{s_{i}}$ be such that $m_{\lambda} \neq 0$ and $n_{s_{i}}-\lambda_{1}=s_{i}, n_{s_{i}}-\lambda_{1}^{\prime} \geq s_{i}$. We construct the partition $\mu=\left(s_{i}, \lambda_{2}, \ldots, \lambda_{r}\right)$ where $\lambda=\left(\lambda_{1}, \ldots, \lambda_{r}\right)$. Since $s_{i}=\sum_{i=2}^{r} \lambda_{i}$ is the number of boxes outside the first row, we have that $\mu \vdash 2 s_{i}$. Let $B$ be a fixed constant with $1<B<2$.

If $\lambda_{2}=1$, then $\mu=\left(s_{i}, 1^{s_{i}}\right) \vdash 2 s_{i} \leq n_{s_{i}}$. Then by the hook formula,

$$
\operatorname{deg} \chi_{\mu}=\left(\begin{array}{c}
2 s_{i}-1 \\
s_{i}
\end{array}\right)>\frac{2^{2 s_{i}}}{2 s_{i}+1} .
$$

Thus there exists $N_{3}>0$ such that for all $i \geq N_{3}$, deg $\chi_{\mu}>B^{2 s_{i}}$. In case $\lambda_{2} \geq 2$, $\mu \leq\left(s_{i}^{s_{i}}\right)$. By Theorem [1] there exists $N_{4}>0$ such that for all $i \geq N_{4}$, $\operatorname{deg} \chi_{\mu}>$ $B^{2 s_{i}}$. Thus in any case, for $i \geq \max \left\{N_{3}, N_{4}\right\}$, we have that $\operatorname{deg} \chi_{\mu}>B^{2 s_{i}}$.

Recall that $n_{s_{i}}=s_{i} a_{s_{i}}$. Hence $s_{i} \geq \frac{n_{s_{i}}}{M}$ and

$$
\operatorname{deg} \chi_{\mu}>B^{2 s_{i}} \geq B^{2 \frac{n_{s_{i}}}{M}}=b^{n_{s_{i}}}
$$


where $b=B^{\frac{2}{M}}>1$ is a constant. Then let $N_{5}>0$ be a constant such that, for all $i \geq \max \left\{N_{3}, N_{4}, N_{5}\right\}, b^{n_{s_{i}}}>C n_{s_{i}}^{k}$. This says that $\operatorname{deg} \chi_{\lambda} \geq \operatorname{deg} \chi_{\mu}>C n_{s_{i}}^{k}$, and we are done.

Therefore we may assume that there exists $N_{6}$ such that, for all $s \geq N_{6}$, we have that $a_{s} \leq 2$. Let $\lambda \vdash n_{s}$ be such that $m_{\lambda} \neq 0$ and $n_{s}-\lambda_{1}=s, n_{s}-\lambda_{1}^{\prime} \geq s$. Since $s a_{s}=n_{s}$, then $\lambda_{1}^{\prime} \leq \lambda_{1}=n_{s}-s \leq n_{s}-\frac{n_{s}}{2}=\frac{n_{s}}{2}$. Thus $\lambda \leq\left(d^{d}\right)$, where $d=\left[\frac{n_{s}}{2}\right]$. By Theorem 1 there exists a constant $N_{7} \geq N_{6}$ such that for all $s \geq N_{7}$, $\operatorname{deg} \chi_{\lambda}>B^{n_{s}}$. Since for some constant $N_{8} \geq N_{7}$ we have that $B^{n_{s}}>C n_{s}^{k}$, we get that for such an $s, \operatorname{deg} \chi_{\lambda}>C n_{s}^{k}$, and the proof is complete.

It is clear that any nilpotent algebra has polynomial growth of the codimensions. Next we give three examples of algebras (associative, Lie and Jordan, respectively) which are not nilpotent but whose codimensions are linearly bounded. Recall that if $\chi_{n}(A)=\sum_{\lambda \vdash n} m_{\lambda} \chi_{\lambda}$ is the $n$-th cocharacter of an algebra $A$, then $l_{n}(A)=\sum_{\lambda} m_{\lambda}$ is called the $n$-th colength of $A$. In each of the three examples, $l_{n}(A)$ will be equal to one for all $n$, and according to [7] these three algebras generate the only varieties whose colength is equal to one.

The first example is $A=F[x]$, the polynomial ring in one variable over $F$. Then $A$ is an associative commutative algebra, and it is easily checked that

$$
\chi_{n}(A)=\chi_{(n)},
$$

for all $n \geq 1$.

The second example is $M_{2}$, the two-dimensional metabelian Lie algebra with basis $h, e$ and multiplication defined by $[h, e]=e$. It is well-known that

$$
\chi_{n}\left(M_{2}\right)=\chi_{(n-1,1)} .
$$

The third example is the algebra $A$ constructed by Shestakov (see [10, Example 2, pg. 84]). Let $V$ be an infinite-dimensional vector space with basis $\left\{e_{1}, e_{2}, \ldots\right\}$ and let $G=\left\{e_{1}, e_{2}, \ldots \mid e_{i} e_{j}=-e_{j} e_{i}\right\}$ be the exterior algebra on $V$ without unit element. We consider the algebra $A=G \oplus V$, with multiplication defined by the rule

$$
(u+x)(v+y)=v x+u y,
$$

where $u, v \in G, x, y \in V$. It is easy to see that $A$ is a Jordan algebra satisfying the identities

$$
x x x \equiv 0,\left(x_{1} x_{2}\right)\left(x_{3} x_{4}\right) \equiv 0 .
$$

Moreover it can be checked that

$$
\chi_{n}(A)=\chi_{\left(2,1^{n-1}\right)} .
$$

It is worth noticing that the set of left normed multilinear monomials $x_{n} x_{i} x_{i_{1}} \ldots$ $x_{i_{n-2}}$, where $i_{1}<\cdots<i_{n-2}$ for $i=1, \ldots, n-1$, is a basis of $P_{n}\left(\bmod \operatorname{Id}\left(M_{2}\right)\right)$ and $(\bmod \operatorname{Id}(A))$.

Due to the well-known duality between $S_{n}$-representations and polynomial $G L$ representations, in the following examples we shall use $G L$-representations, and we refer the reader to [2] for an account of the theory.

Next we give an example of an algebra $A$ satisfying condition (2) of the theorem but not condition (1), and $c_{n}(A)$ is not polynomial bounded.

Example 1. Let $A$ be the algebra generated by one element $a$ such that every word in $A$ containing two or more subwords equal to $a^{2}$ must be zero. 
Let $\lambda \vdash n$ be such that $n-\lambda_{1} \geq 2$. Then if $f\left(x_{1}, \ldots, x_{n}\right)$ is a highest weight vector associated to $\lambda, f$ is alternating either on three variables or on two pairs of variables. In either case every monomial of $f$ evaluates to a word of $A$ containing at least two subwords equal to $a^{2}$. It follows that $f \in \operatorname{Id}(A)$, and so the corresponding multiplicity $m_{\lambda}$ in $\chi_{n}(A)$ must be zero. Thus

$$
\chi_{n}(A)=m_{(n)} \chi_{(n)}+m_{(n-1,1)} \chi_{(n-1,1)} .
$$

Let $L_{a}$ and $R_{a}$ denote the linear transformations on $A$ of left and right multiplication by $a$, respectively. If $w\left(L_{a}, R_{a}\right)$ is any word of length $n-2$ in $L_{a}$ and $R_{a}$, then $w\left(L_{a}, R_{a}\right)\left(a^{2}\right)$ is the evaluation of a highest weight vector associated to the partition $(n)$ which is not an identity of $A$. Since there are $2^{n-2}$ distinct such words, we get $2^{n-2}$ highest weight vectors which are linearly independent $\bmod \operatorname{Id}(A)$. Thus $m_{(n)}=2^{n-2}$ and $c_{n}(A) \geq 2^{n-2}$ is not polynomial bounded.

If $A$ is an associative algebra, an equivalent condition for the codimensions of $A$ to be polynomially bounded is that its colength is bounded by a constant, i.e., $l_{n}(A)=\sum_{\lambda \vdash n} m_{\lambda}<K$, for some constant $K$. Unfortunately this is not true for general nonassociative algebras or even for Lie algebras.

In fact, as the referee has pointed out (see [1]), one may fix a positive integer $d$ and consider the Lie T-ideal generated by all multilinear Lie polynomials which generate irreducible $S_{n}$-modules with $n-\lambda_{1}>d$. Then the $S_{n}$-cocharacter is a sum of all irreducible $S_{n}$-characters with $n-\lambda_{1} \leq d$ with multiplicity equal to the multiplicity in the free Lie algebra. Even for $d=2$ one has colength approximately equal to $n$, since in the free Lie algebra $m_{(n-1,1)}=1, m_{(n-2,2)}=\left[\frac{n-3}{2}\right]$ and $m_{(n-2,1,1)}=\left[\frac{n-2}{2}\right]$. For $d$ large enough the colength may grow as a polynomial of an arbitrarily high degree.

We next give one more example of an algebra $A$ such that $\left\{c_{n}(A)\right\}_{n \geq 1}$ is polynomial bounded, hence satisfies conditions (1) and (2) of Theorem 2. but $m_{(n)}$ grows polynomially like $\frac{n}{2}$.

Example 2. Let $A$ be the commutative algebra generated by one element $a$ such that $\left(a^{i} a^{j}\right) a^{k}=0$ for all $i \geq j \geq 2$ and $k \geq 1$. Here $a^{t}=(((a a) a) \cdots a)$ is the left normed product of $a$ with itself $t$ times.

We claim that if $\lambda \vdash n$ is such that $n-\lambda_{1} \geq 3$, then $m_{\lambda}=0$. In fact, if $f\left(x_{1}, \ldots, x_{n}\right)$ is a highest weight vector associated to $\lambda$, every monomial of $f$ evaluates to a word of $A$ containing some $a^{i} a^{j} a^{k}$ with $i \geq j \geq 2$ and $k \geq 1$. Thus $f \in \operatorname{Id}(A)$, and condition (1) is satisfied.

Now if we consider all arrangements of brackets on a word in $A$ of length $n$, we get the words $a^{n}$ and $a^{i} a^{j}$ with $i \geq j \geq 2, i+j=n$. Their number is $\left[\frac{n}{2}\right]$. Hence if $m$ is defined as in condition (2) of Theorem 2, we have that $m \leq \frac{1}{2} n$.

We remark that this also implies that there are $\left[\frac{n}{2}\right]$ highest weight vectors associated to the partition $(n)$ which are linearly independent $\bmod \operatorname{Id}(A)$. Thus $\left[\frac{n}{2}\right]$ is an upper bound for the multiplicity $m_{(n)}$.

\section{REFERENCES}

[1] I. I. Benediktovich and A. E. Zalesskiı̌, T-ideals of free Lie algebras with polynomial growth of a sequence of codimensionalities (Russian), Vestsī Akad. Navuk BSSR Ser. Fīz-Mat. Navuk, 1980, 3, 5-10. MR 582766 (81k:17002)

[2] V. Drensky, "Free Algebras and PI-Algebras, Graduate Course in Algebra", Springer-Verlag Singapore, Singapore, 2000. MR1712064(2000j:16002) 
[3] A. Giambruno and M. Zaicev, "Polynomial Identities and Asymptotic Methods", Mathematical Surveys and Monographs, Vol. 122, Amer. Math. Soc., Providence, RI, 2005. MR 2176105 (2006g:16054)

[4] G. James and A. Kerber, "The representation theory of the symmetric group", Encyclopedia of Mathematics and its Applications, Vol. 16, Addison-Wesley, Reading, MA, 1981. MR644144(83k:20003)

[5] A. R. Kemer, T-ideals with power growth of the codimensions are Specht (Russian), Sibirskii Matematicheskii Zhurnal 19 (1978), 54-69; English translation: Siberian Math. J. 19 (1978), 37-48. MR0466190 (57:6070)

[6] S. P. Mishchenko, Lower bounds on the dimensions of irreducible representations of symmetric groups and of the exponents of the exponential of varieties of Lie algebras, Russian summary, Sb. Math. 187 (1996), No. 1, 83-94; translation from Mat. Sb. 187 (1996), No. 1, 81-92. MR 1380205 (97d:17003)

[7] S. Mishchenko, Varieties with colength equal to one (Russian), Vest. Mosk. Univ., Ser. I-Mat. (to appear).

[8] A. Regev, Existence of identities in $A \otimes B$, Israel J. Math. 11 (1972), 131-152. MR0314893 $(47: 3442)$

[9] I. B. Volichenko, Varieties of Lie algebras with identity $\left[\left[X_{1}, X_{2}, X_{3}\right],\left[X_{4}, X_{5}, X_{6}\right]\right]=0$ over a field of characteristic zero (Russian), Sibirsk. Mat. Zh. 25 (1984), 3, 40-54. MR746940 (85k:17016)

[10] K. A. Zhevlakov, A. M. Slin'ko, I. P. Shestakov, A. I. Shirshov, "Rings That Are Nearly Associative", Pure and Applied Mathematics, Vol. 104, Academic Press, Inc. [Harcourt Brace Jovanovich, Publishers], New York-London, 1982. MR668355 (83i:17001)

Dipartimento di Matematica e Applicazioni, Università di Palermo, Via Archirafi 34, 90123 Palermo, Italy

E-mail address: a.giambruno@unipa.it

Department of Algebra and Geometric Computations, Ulyanovsk State University, ULYANOVSK 432970, RUSSIA

E-mail address: mishchenkosp@mail.ru 